\title{
Plasma Erythropoietin, IL-17A, and IFNy as Potential Biomarkers of Motor Function Recovery in a Canine Model of Spinal Cord Injury
}

\author{
Lijian Zhang ${ }^{1,2,3}$ (D) $\cdot$ Xiaoqing Zhuang ${ }^{4}$ (D) $\cdot$ Yao Chen ${ }^{1,2,3} \cdot$ Zhanfeng Niu $^{2} \cdot$ Hechun Xia $^{2,3}$
}

Received: 24 February 2020 / Accepted: 30 April 2020 / Published online: 16 May 2020

(C) The Author(s) 2020

\begin{abstract}
Traumatic spinal cord injury (SCI) is a devastating neurological disease for which an accurate, cost-effective prediction of motor function recovery is in pressing need. A plethora of neurochemical changes involved in the pathophysiological process of SCI may serve as a new source of biomarkers for patient outcomes. Five dogs were included in this study. We characterized the plasma cytokine profiles in acute phase $(0,1$, and 3 days after SCI) and subacute phase $(7,14$, and 21 days after SCI) with microarray analysis. The motor function recovery following SCI was monitored by Olby scores. The expression level of differentially expressed proteins (DEPs) was measured with enzyme-linked immunosorbent assay (ELISA). Then, correlations with the Olby scores and receiver operating characteristic curve (ROC) analysis were performed. We identified 12 DEPs including 10 pro-inflammatory and 2 anti-inflammatory cytokines during the 21-day study period. Among those, the expression levels of erythropoietin (EPO), IL-17A, and IFN $\gamma$ significantly correlated with the Olby scores with $R^{2}$ values of $0.870,0.740$, and 0.616 , respectively. The results of the ROC analysis suggested that plasma EPO, IL-17A, and IFN $\gamma$ exhibited a significant predictive power with an area under the curve (AUC) of $0.656,0.848$, and 0.800 for EPO, IL-17A, and IFN $\gamma$, respectively. Our results provide a longitudinal description of the changes in plasma cytokine expression in the acute and subacute stages of canine SCI. These data reveal novel panels of inflammation-related cytokines which have the potential to be evaluated as biomarkers for predicting motor function prognosis after SCI.
\end{abstract}

Keywords Spinal cord injury $\cdot$ Plasma $\cdot$ Inflammatory cytokines $\cdot$ Motor function $\cdot$ Biomarkers

\section{Introduction}

Traumatic spinal cord injury (SCI) typically results in partial or substantial loss of motor function below the level of injury site,

Electronic supplementary material The online version of this article (https://doi.org/10.1007/s12031-020-01575-y) contains supplementary material, which is available to authorized users.

Xiaoqing Zhuang

zhuangxq@nyfy.com.cn

$\triangle$ Hechun Xia

xhechun@nyfy.com.cn

1 School of Clincial Medicine, Ningxia Medical University, Yinchuan, Ningxia, China

2 Department of Neurosurgery, General Hospital of Ningxia Medical University, No. 804, Shengli Street, Xingqing District, Yinchuan, Ningxia, China

3 Ningxia Human Stem Cell Research Institute, General Hospital of Ningxia Medical University, Yinchuan, Ningxia, China

4 Department of Nuclear Medicine, General Hospital of Ningxia Medical University, No. 804, Shengli Street, Xingqing District, Yinchuan, Ningxia, China which decreases the quality of life of individuals with SCI (Kumar et al. 2018). Approximately 8000 thousand people will suffer this catastrophic injury each year throughout the world (Gerasimenko et al. 2015). Recently, substantial evidences have shown that the initial neurological examination is extremely difficult for predicting long-term outcomes (Rodrigues et al. 2018; Rasmussen and Carlsen 2016). Even though the injury may seem the same in type and severity, patients could have heterogeneous recovery and uncertain prognosis (Anderson 2004; Raineteau and Schwab 2001). Obtaining accurate and reliable prediction of motor functional improvement is crucial for neurosurgeons and rehabilitation teams to optimize the individualized neuro-restorative treatment options. Therefore, identification of valuable biomarkers of SCI remains a worthy task.

Neurochemical biomarkers have long held promise in the field of SCI to monitor the progression of its pathology and to predict treatment responses longitudinally (Hulme et al. 2017). These biomarkers in general represent molecules released from damaged cells and the acute inflammatory response (acute biomarkers), molecules released during the cellular and immunological response while healing the injury (subacute biomarkers) (Kwon et al. 2019). As peripheral blood is easily accessible 


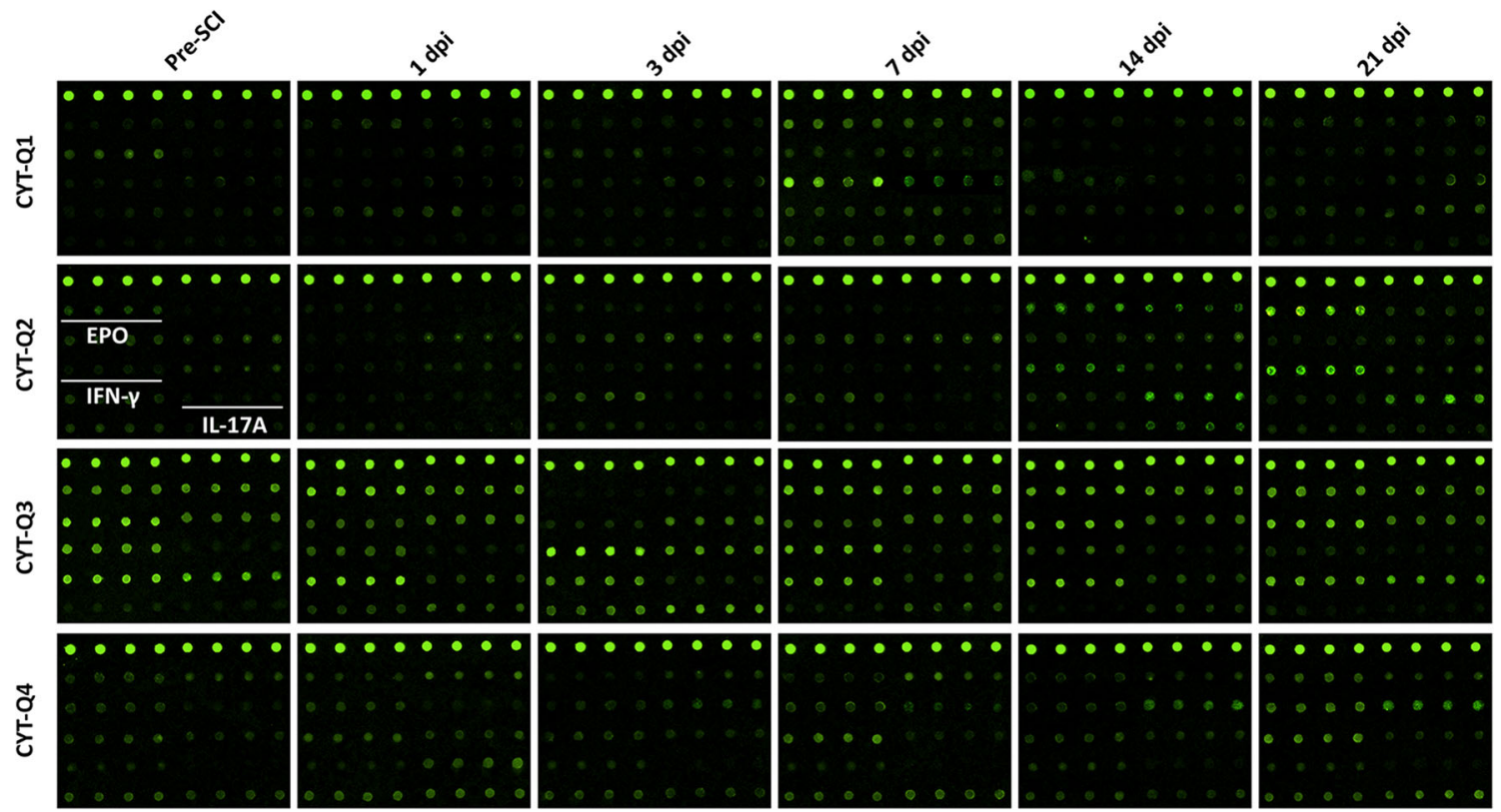

Fig. 1 Fluorescence images of microarray chips. The location of EPO, IFN $\gamma$, and IL-17A in the array is labeled by white underscores. The fluorescence intensity of the spots indicates the level of expression

and requires less invasive procedures, it was widely used in both animal studies and clinical trials for biomarker screening (Wang et al. 2019). Such protein markers, including tumor necrosis factor- $\alpha(\mathrm{TNF}-\alpha)$ and interleukins (ILs), were identified in previous studies (Yousefifard et al. 2019). To date, the vast majority of studies that aimed to discovery plasma/serum biomarkers for $\mathrm{SCI}$ have been carried out in preclinical rodent models. However, some hypothesis-driven clinical trials based on rodent results have disappointingly failed, which might be explained by the potential differences between humans and experimental animals (Kwon et al. 2010). Before translating promising findings obtained from the laboratories into clinical settings, preclinical validation of the efficacy in large-animal models is needed. Dogs serve as a good intermediate biological model, bridging the large gap between human and rat studies, as dogs not only have heterogeneity of both injury and genetic backgrounds but also have histopathologic similarities to those in human patients (Dalgaard 2015; Jeffery et al. 2005).

Systemic inflammation plays a pivotal role in promoting many of the pathological consequences of SCI. Previous studies have demonstrated that inflammation-related responses may be a feasible indicator for predicting the prognosis and severity of SCI (Yang et al. 2005; Kijima et al. 2019). Therefore, a greater insight into the molecular dynamics

Table 1 The list of differentially expressed cytokines (pg/ml)

\begin{tabular}{|c|c|c|c|c|c|c|}
\hline & Pre-SCI & $1 \mathrm{dpi}$ & 3 dpi & 7 dpi & 14 dpi & $21 \mathrm{dpi}$ \\
\hline IL-10 & $74.04 \pm 41.92$ & $20.67 \pm 19.14 * * * *$ & $83.66 \pm 34.80$ & $130.09 \pm 49.57$ & $176.79 \pm 120.53$ & $74.38 \pm 40.19$ \\
\hline EPO & $1011.78 \pm 830.46$ & $1009.3 \pm 666.14$ & $552.78 \pm 188.21$ & $522.78 \pm 250.49$ & $7613.48 \pm 1308.58 * * * *$ & $9146.79 \pm 700.23 * * * *$ \\
\hline MIP $1 \beta$ & $65.39 \pm 60.59$ & $49.29 \pm 30.43$ & $38.55 \pm 26.19$ & $59.96 \pm 22.60$ & $849.71 \pm 109.22 * * * *$ & $1062.1 \pm 207.89 * * * *$ \\
\hline GM-CSF & $9.41 \pm 6.77$ & $11.36 \pm 14.67$ & $30.89 \pm 14.11$ & $50.40 \pm 16.31 * *$ & $37.38 \pm 16.83$ & $21.10 \pm 11.84$ \\
\hline GASP 1 & $2082.21 \pm 564.18$ & $1834.3 \pm 224.79$ & $1202.59 \pm 266.79^{*}$ & $2080.71 \pm 639.20$ & $2582.31 \pm 186.45$ & $2692.17 \pm 282.30$ \\
\hline FGF7 & $38.87 \pm 48.56$ & $43.87 \pm 59.20$ & $34.84 \pm 20.52$ & $25.24 \pm 11.72$ & $153.34 \pm 70.31 *$ & $105.40 \pm 50.63$ \\
\hline TNF R1 & $4.55 \pm 9.10$ & $1.79 \pm 3.57$ & $10.61 \pm 18.01$ & $11.53 \pm 23.06$ & $279.21 \pm 163.72 * * *$ & $197.44 \pm 108.16^{*}$ \\
\hline NOPE & $4242.31 \pm 1871.29$ & $1763.04 \pm 628.61$ & $1652.22 \pm 1186.37^{*}$ & $2735.73 \pm 839.79$ & $2938.60 \pm 1039.98$ & $3439.99 \pm 963.74$ \\
\hline $\mathrm{IFN} \gamma$ & $1.76 \pm 3.52$ & $38.29 \pm 15.31$ & $0.00 \pm 0.00$ & $0.00 \pm 0.00$ & $537.70 \pm 14.19 * * * *$ & $584.86 \pm 56.55^{* * * * *}$ \\
\hline IL-1 $\alpha$ & $52.01 \pm 61.16$ & $23.54 \pm 17.05$ & $51.48 \pm 44.15$ & $26.81 \pm 20.17$ & $394.88 \pm 109.51 * * * *$ & $522.24 \pm 110.69 * * * *$ \\
\hline IL-1 $\beta$ & $91.82 \pm 55.51$ & $121.18 \pm 23.85$ & $149.08 \pm 116.42$ & $86.20 \pm 56.11$ & $177.42 \pm 17.38$ & $310.04 \pm 146.17^{*}$ \\
\hline IL-17A & $13.32 \pm 24.18$ & $18.61 \pm 22.19$ & $8.93 \pm 10.06$ & $21.65 \pm 36.92$ & $394.73 \pm 404.11$ & $560.61 \pm 282.52 * *$ \\
\hline
\end{tabular}

Compared with baseline expression level of pre-SCI dogs. Data are presented as mean $\pm \mathrm{SD}, n=5$ in each group, $* p<0.05, * * p<0.01$, *** $p<0.001$, $* * * * p<0.0001$ 
Fig. 2 The temporal profile of plasma cytokines following SCI. a Heat map analysis of following SCI in dogs. Color bar: green indicates downregulation; red indicates upregulation. b Temporal expression of the selected pro- and antiinflammatory cytokines in plasma of SCI dogs: EPO, IFN $\gamma$, IL-17A, IL- $1 \beta$, and IL- $1 \alpha$. Data are presented as mean $\pm \mathrm{SD}, n=5$ in each group, $* p<0.05$, $* * * * p<0.0001$ differentially expressed cytokines

a

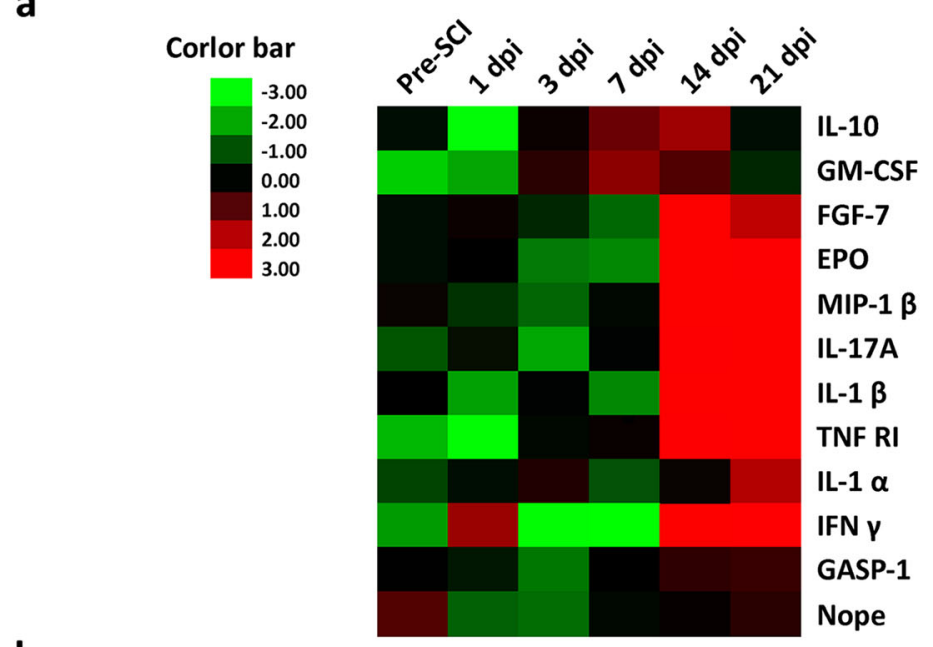

b
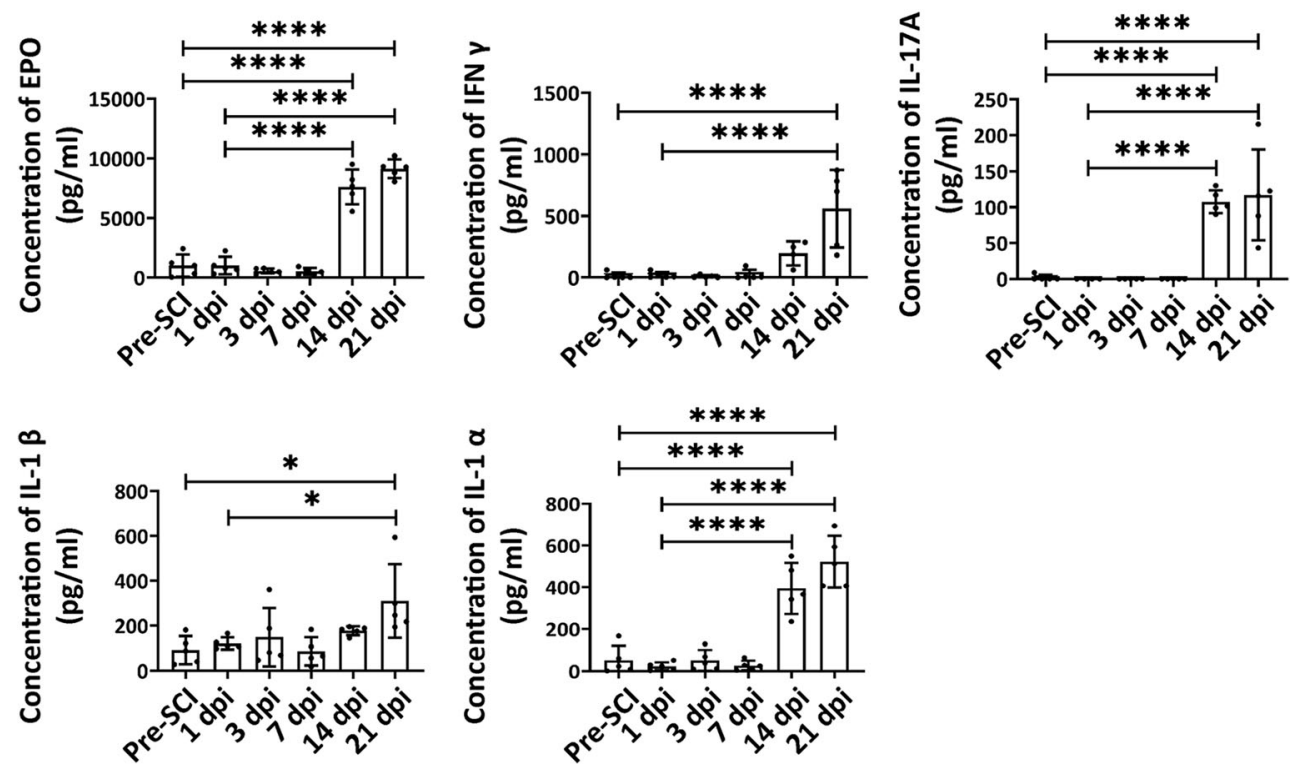

Pharmaceutical Co., Ltd., Shijiazhuang, China; $2.5 \mathrm{mg} / \mathrm{kg}$, intramuscularly) was given to the dogs $30 \mathrm{~min}$ before the operation. The operation was carried out under general anesthesia with 2.0 $3.0 \%$ isoflurane in oxygen. During the operation, body temperature was maintained using a heating pad. Using sanitized instruments, laminectomy was performed at the tenth thoracic segment (T10), followed by a unilateral left side hemisection at the T10 level, which was performed by micro-scissors. Before voluntary urination establishment, manual bladder expression was performed at least three times a day.

\section{Protein Antibody Array}

A total of 5 healthy vaccinated 12-24-month-old beagle dogs weighing $13-17 \mathrm{~kg}$ were used in this study. All surgical procedures followed the standards and guidelines set out by the Experimental Animal Centre of Ningxia Medical University (2017-073). The painkiller (tramadol hydrochloride, CSPC
Plasma samples $(5 \mathrm{ml})$ were collected from the jugular vein of each dog for cytokine analysis at 0 day (pre-SCI), 1 day, 3 days, 7 days, 14 days, and 21 days post-injury (dpi). According to the manufacturer's instructions, plasma samples 


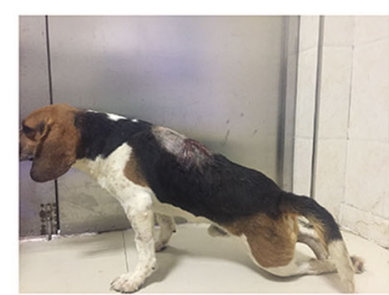

Pre-SCl

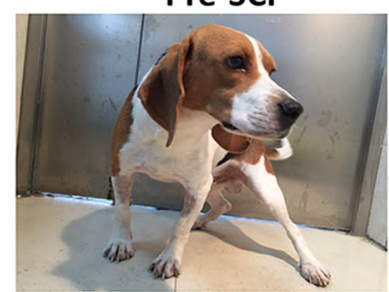

$14 \mathrm{dpi}$

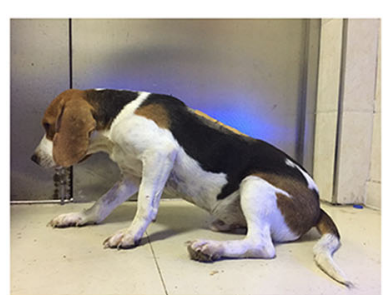

$7 \mathrm{dpi}$

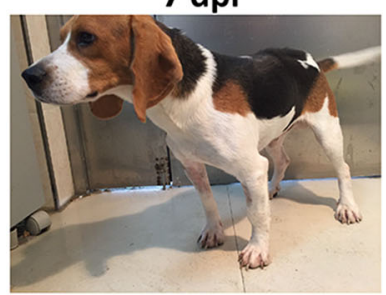

21 dpi

b

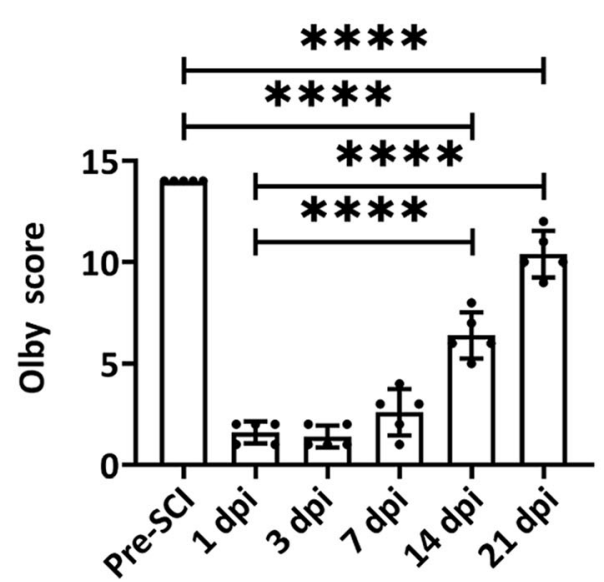

Fig. 3 Motor function assessments with Olby score. a Representative images of motor functional recovery of SCI dogs. b Changes in the Olby scores during the 21-day study period. Data are presented as mean $\pm \mathrm{SD}, n=5$ in each group, $* * * * p<0.0001$

and standard curve cocktails were incubated on a Quantibody Canine Cytokine Array Q1/2/3/4 (RayBiotech, Norcross, GA, USA), which simultaneously detects 40 cytokines (details in Supplementary Table S1). After the experimental procedures, the glass slides were scanned to detect the fluorescent signals in the microarray, and the signals were visualized using a GenePix Professional 4200A scanner (Molecular Devices, Sunnyvale, CA, USA). Data extraction was performed using GenePix Pro 5.1 software. Cytokine levels were normalized to milligrams of tissue.

\section{Cytokine Measurement with Enzyme-Linked Immunosorbent Assay}

The plasma levels of EPO, IL-17A, and IFN $\gamma$ were measured by enzyme-linked immunosorbent assay (ELISA) (RayBiotech, Norcross, GA, USA) according to the manufacturer's instructions. In brief, protein extracts of the plasma samples were incubated in plates coated with the capture

antibody overnight at $4{ }^{\circ} \mathrm{C}$. The plates were washed, and a biotin-conjugated detection antibody was added to the plates and incubated at room temperature for $2 \mathrm{~h}$ to bind to the corresponding proteins. HRP-conjugated streptavidin was added to the plates and allowed to incubate for $45 \mathrm{~min}$. Tetramethylbenzidine dihydrochloride (TMB) reagent was added and allowed to incubate for $30 \mathrm{~min}$ before the reaction was stopped with sulfuric acid. The optical density was measured via an ELx800NB microplate reader (BioTek, Winooski, CT, USA) at a wavelength of $450 \mathrm{~nm}$.

\section{Motor Function Recovery Assessment}

All dogs were subjected to motor function assessment to exclude any motor deficits before the experiment. After the establishment of SCI model, the dogs were observed for any spontaneous recovery of motor function using Olby score (Olby et al. 2001) in the acute phase (0, 1, and 3 dpi) and the subacute phase (7, 14, and $21 \mathrm{dpi}$ ) (Moore et al. 2017).

\section{Statistical Analysis}

All data were statistically analyzed by using the GraphPad Prism (version 7.0, GraphPad Software Inc., San Diego, CA, USA) with nonparametric Kruskal-Wallis test followed by Dunnett's multiple comparison. Correlation analysis was performed using Pearson's product moment $(R)$ correlation. The area under the receiver operating characteristic (ROC) curve was performed for predictive power estimation. All values are presented as the mean \pm standard deviation (SD). $p<0.05$ was considered statistically significant.

\section{Results}

\section{Cytokine Profiling in Plasma}

In this study, we investigate the temporal changes in plasma cytokines obtained from SCI dogs with protein chip array (Fig. 1). Heat map analyses showed significant changes in the concentration of 10 pro-inflammatory cytokines (GM-CSF, GASP-1, FGF7, TNF R1, NOPE, IL- $1 \alpha$, IL-1 $\beta$, IFN $\gamma$, MIP$1 \beta$, and IL-17A) and 2 anti-inflammatory cytokines (EPO and IL-10) after SCI (Table 1, Fig. 2a). At 14 and 21 dpi, the concentrations of both IL-17A $(p<0.0001, n=5 ; p<0.0001, n=5)$ and IFN $\gamma(p<0.0001, n=5 ; p<0.0001, n=5)$ were significantly elevated. The level of EPO tended to decrease within $7 \mathrm{dpi}$, but without significant difference with pre-SCI level, and then increased markedly at 14 and $21 \mathrm{dpi}(p<0.0001, n=5$; $p<0.0001, n=5$, respectively). And the concentration of IL$1 \alpha$ fluctuated within first 7 dpi with an initially decreased expression on $1 \mathrm{dpi}(p<0.05, n=5)$ that then became significantly elevated on 14 and 21 dpi $(p<0.0001, n=5 ; p<0.0001, n=5)$. 
However, the expression of IL-1 $\beta$ appeared to be biphasic, with two peaks at $3 \mathrm{dpi}$ and $21 \mathrm{dpi}$, and the significant changes occurred at 21 dpi ( $p=0.40, n=5 ; p<0.0001, n=5$, respectively) (Fig. 2b).

\section{Assessment of Motor Function}

The motor function of SCI dogs was assessed using the Olby score. As shown in Fig. 3, all dogs showed an Olby score of 1, suggesting no motor function in the left lower limb after the establishment of SCI. Gradual recovery of motor function in the paralyzed lower limb was observed in all dogs after SCI. The Olby score reached significance at $14 \mathrm{dpi}$ and $21 \mathrm{dpi} \mathrm{com-}$ pared with that at $1 \mathrm{dpi}(6.40 \pm 1.02$ vs $1.60 \pm 0.49, p<0.0001$, $n=5 ; 10.04 \pm 1.01$ vs $1.60 \pm 0.49, p<0.0001, n=5$ ).

\section{Plasma EPO, IFN $\gamma$, and IL-17A Concentrations for Motor Functional Prognosis}

To validate the expression level of EPO, IFN $\gamma$, and IL$17 \mathrm{~A}$, the ELISA analysis was performed. In accordance with the results obtained from the Quantibody Cytokine Array, the ELISA experiments showed that IL-17A, IFN $\gamma$, and EPO were significantly upregulated at 14 and 21 dpi $(p<0.0001, n=5 ; p<0.0001, n=5$; and $p<0.0001, n=5$, respectively) (Fig. $4 \mathrm{a}-\mathrm{c}$ ).
To investigate whether the concentration of EPO, IFN $\gamma$, and IL-17A could serve as biomarkers of motor function recovery, we next performed a linear regression analysis. Our results showed that the concentration of EPO $\left(R^{2}=0.8023\right.$, $p<0.0001)$, IFN $\gamma\left(R^{2}=0.6549, p<0.0001\right)$, and IL-17A $\left(R^{2}=0.7331, p<0.0001\right)$ correlated well with the Olby score (Fig. 4d-f).

\section{ROC Analysis}

The ROC curve of the cytokines reflected separation between SCI dogs and dogs without SCI, with an area under the curve (AUC) of $0.656,0.848$, and 0.800 for EPO, IL-17A, and IFN $\gamma$, respectively (Fig. 5). Then, we compared the specificity and sensitivity for each plasma protein individually (Table 2).

\section{Discussion}

To date, several research groups have reported poor feasibility and accuracy of current methods in classifying injury severity and predicting neurologic outcomes due to the heterogeneity of SCI or limitations of each imaging technology (Krishna et al. 2014; Hicks et al. 2017). For example, Kaminski et al. (2017) created a prediction model including four different acute clinical parameters associated with an $R^{2}$ value of 0.573 . Here, we investigated the temporal changes in the a
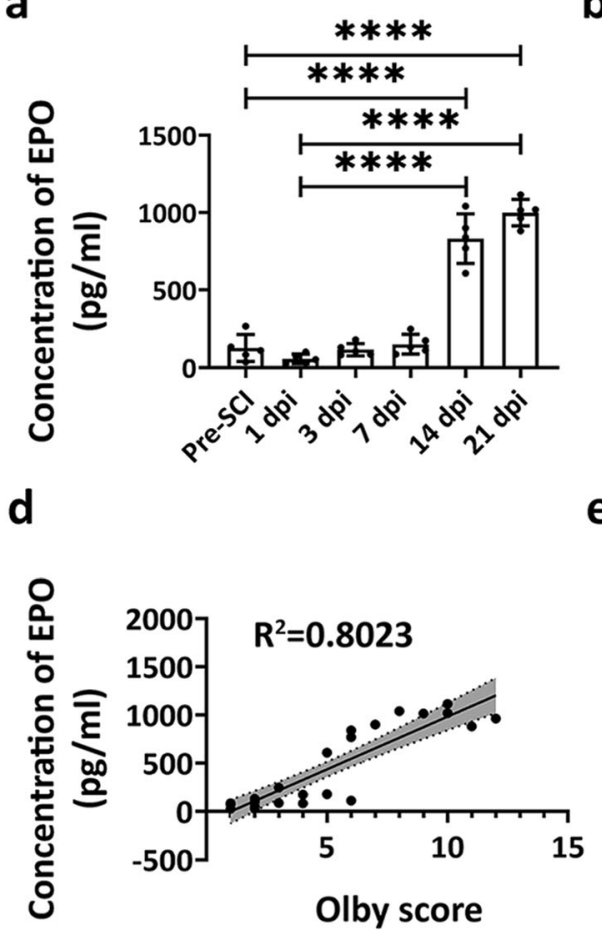

Fig. 4 ELISA validation for EPO, IFN $\gamma$, and IL-17A and correlation with the Olby score. The concentration of EPO (a), IFN $\gamma(\mathbf{b})$, and IL-17A (c) obtained from ELISA are shown by scatter plot. Correlation between the b
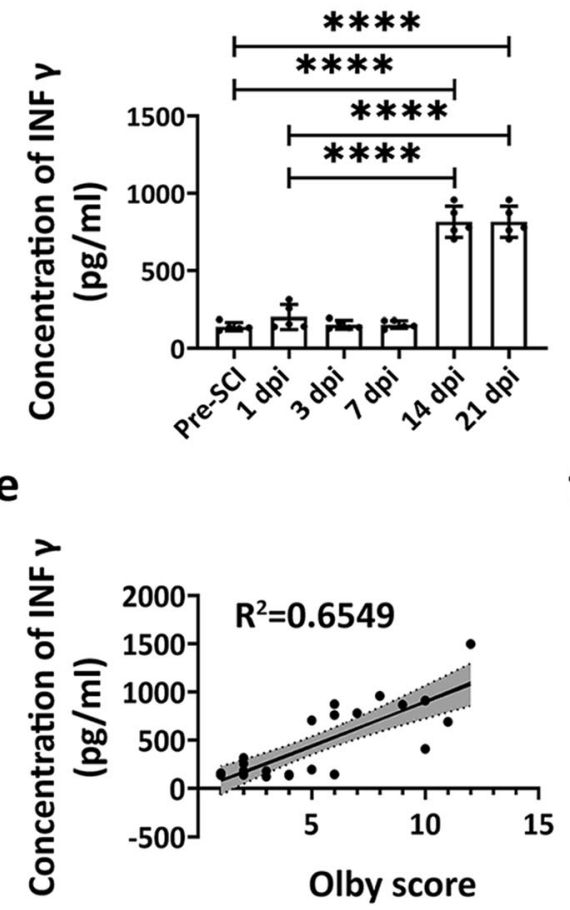

C
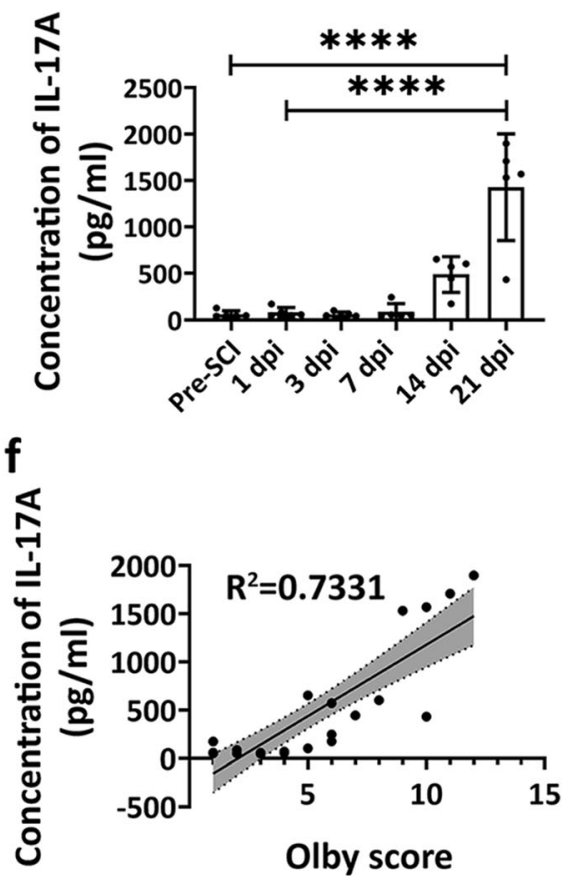

Olby score and EPO (d), IFN $\gamma(\mathbf{e})$, and IL-17A (f) concentrations. Data are presented as mean $\pm \mathrm{SD}, n=5$ in each group, $* * * * p<0.0001$ 
expression level of 40 cytokines using cytokine arrays. Our results showed that inflammation-related cytokines exhibited improved predictive power with $R^{2}$ values of 0.870 (EPO), 0.740 (IFN $\gamma$ ), and 0.616 (IL-17A) in acute and subacute stages of SCI. This indicates that plasma EPO, IL-17A, and IFN $\gamma$ could be potential biomarkers for motor functional prognosis in the acute and subacute phases of SCI.

EPO is a robust biomarker of motor functional recovery identified in our current study. Until now, a substantial number of animal models and clinical trials have shown the efficiency of both endogenous and exogenously administered EPO for neurological recovery (Costa et al. 2015; Carelli et al. 2017; Grasso et al. 2005). Little is known about its potential to serve as a biomarker for SCI. To the best of our knowledge, this is the first study investigating the correlation of endogenous levels of EPO with motor function following SCI. Previous studies have reported that the release of pro-inflammatory cytokines or metabolic stress induced by tissue injury could activate EPO production (Carelli et al. 2011). And EPO could protect a wide variety of cells and tissues in turn, from apoptosis induced by hypoxia, as well as from excitotoxins and glucose deprivation following SCI (Matis and Birbilis 2009; Foley et al. 2017). Evidence has shown that the plasma EPO is significantly increased in SCI patients, returning to normal after 8 weeks (Claus-Walker and Dunn 1984). As a tissue-protective cytokine, the neuroregenerative effects of EPO have been extensively investigated in various species SCI model (Celik et al. 2002; Simon et al. 2011; Simon et al. 2016). EPO reduced astrogliosis (Vitellaro-Zuccarello et al. 2008) and scar formation (Gorio et al. 2005) and enhanced remyelination by promoting oligodendrogenesis (Cho et al. 2012), thus improving functional neurological status after SCI. The findings obtained from Hong et al.'s study also demonstrated that EPO could increase neurite outgrowth after SCI. Spinal cord neurons grown in the absence of EPO were shorter and fewer in number compared with those grown in the presence of EPO (Hong et al. 2018). Such benefits of neuroprotective activity may underlie the correlation of EPO with motor function recovery following SCI, as was observed in this study.

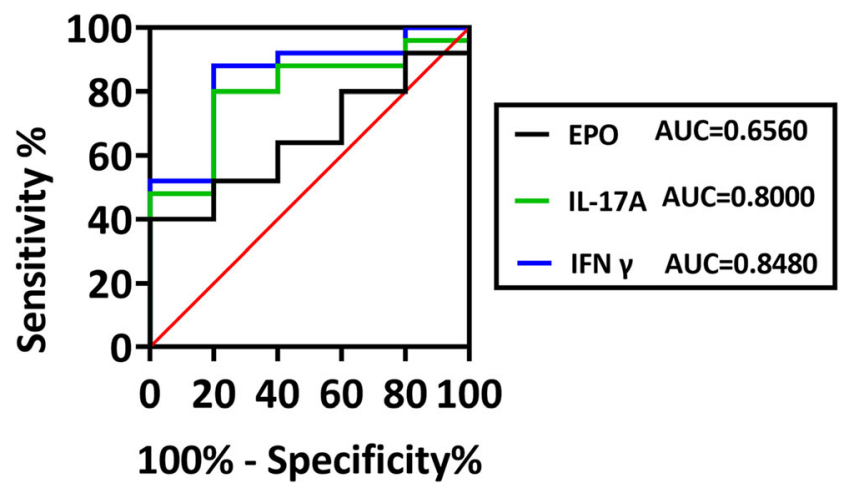

Fig. 5 Receiver operating characteristic (ROC) curve analysis. ROC curve analysis of EPO, IL-17A, and IFN $\gamma$ for determining their prognostic abilities. AUC, area under the ROC curve
Among the differentially expressed proteins (DEPs), the expression level of IL-17A and IFN $\gamma$ was also positively correlated with the motor function. Substantial studies suggest that IL-17A is not only exclusively a pro-inflammatory factor but also has a potential role in neuroanatomical plasticity (Moynes et al. 2014; Chisholm et al. 2012; Habash et al. 2015). The results obtained from $\mathrm{Hu}$ et al.'s study showed that the high level of IL-17A treatment could protect neurons from apoptosis via the activation of JAK2/STAT-3 signaling and the suppression of voltage-dependent $\mathrm{Ca}^{2+}$ influx. And the blockade of IL$17 \mathrm{~A}$ function at late phases of experimental autoimmune uveoretinitis (EAU) resulted in significantly neural cell apoptosis and tissue damage (Hu et al. 2014). Another study also suggested that the high dose of IL-17A administration exerts anti-apoptotic and neuroprotective activity via miR-155-5p downregulation (Ksiazek-Winiarek et al. 2017). Similarly, IFN $\gamma$ is also a pleiotropic cytokine that exhibits both detrimental and neuroprotective effects. Increasing evidences have indicated the beneficial aspects of upregulated IFN $\gamma$ in SCI. Fujiyoshi and his colleagues (Fujiyoshi et al. 2010) showed that the intraperitoneal administration of IFN $\gamma$ significantly facilitated the recovery of motor function with reduced inflammatory cells concentrated in the injury site following SCI. In addition, $\mathrm{SCI}$ applied to IFN $\gamma \mathrm{KO}$ mice resulted in a greater proportion of degenerating neurons in the ventral horn (Victório et al. 2010). IFN $\gamma$ also can activate the choroid plexus to boost the recruitment of inflammation-resolving cells such as T cells and monocyte-derived macrophages which support functional recovery following SCI (Kunis et al. 2013). Moreover, Ishii et al. (2013) suggested that IFN $\gamma$ contributes to the upregulation of glial cell line-derived neurotrophic factor (GDNF) and the secretion of IL-10 in Th1 cells, which might have positive effects on neuroprotection after SCI. And the transfer of IFN $\gamma$ producing Th1 cells could enhance axonal remodeling of corticospinal tract and serotonergic fibers, and promote the functional recovery in SCI mice (Ishii et al. 2012). In light of the above literatures, we proposed that the improved motor function observed in our study might be related to their neuroprotective effects. Histological examination of spinal cord tissue that reveals the neuroanatomical changes should be included in our future work.

Other identified DEPs, such as IL- $1 \alpha$ and IL- $1 \beta$, which were reported to be potential biomarkers for SCI (Yokobori et al. 2015), did not reach any statistical significance

Table 2 Comparison of the predictive abilities of the selected cytokines

\begin{tabular}{lllll}
\hline & Sensitivity (\%) & Specificity (\%) & AUC & $95 \%$ CI \\
\hline EPO & 40 & 100 & 0.656 & 23.40 to 59.26 \\
IFN $\gamma$ & 88 & 80 & 0.848 & 70.04 to 95.83 \\
IL-17A & 80 & 80 & 0.800 & 60.87 to 91.14 \\
\hline
\end{tabular}

$A U C$, area under the ROC curve 
correlation with the motor function. Moreover, the IL- $1 \beta$ detection in blood is in a very limited period of time, because the increased IL-1 $\beta$ can be found mainly during a time when no leucocytosis appears (Christian et al. 2013).

However, this study has limitations. First, the sample size of this study is small and the available protein antibody arrays for canine study are limited. A larger and prospective cohort study with different severity of SCI canines should be conducted to validate our results in the future. Second, this study is only focused on the acute and subacute stages of SCI. It would be also very important to investigate the alternations in the expression level of cytokines and their correlation with motor function in the chronic phase of SCI.

\section{Conclusion}

Our findings provided a comprehensive description of dynamic cytokine profiles in the acute and subacute stages of SCI canines. In this longitudinal study, we identified panels of inflammation-related biomarker candidates including EPO, IFN $\gamma$, and IL-17A with high potential to predict motor function prognosis following the acute and subacute phase of SCI.

\begin{abstract}
Authors' Contributions The work presented here was carried out in collaboration of all authors. H.X. and X.Z. designed the study and reviewed and edited the manuscript. L.Z. performed the experiments, collected the data, performed the statistical analysis, and drafted the manuscript. Y.C. performed the experiments and data collection. Z.N. performed the data analysis and interpretation. All authors read and approved the final manuscript.
\end{abstract}

Funding Information This work was funded by the Key Research Projects of the Ningxia Hui Autonomous Region (Grant No. 2018BCG01002) and the Natural Science Fund of the Ningxia Hui Autonomous Region (Grant No. 2018AAC02012).

\section{Compliance with Ethical Standards}

Ethics Approval and Consent to Participate All animal experiments were approved by the Experimental Animal Centre of Ningxia Medical University (2017-073). National guidelines for the care and use of laboratory animals were followed in animal protocols and experimental procedures described in this article.

Conflict of Interest The authors declare that they have no conflict of interest.

Abbreviations $D E P S$, differentially expressed proteins; $E P O$, erythropoietin; $T N F-\alpha$, tumor necrosis factor- $\alpha$; ILs, interleukins; $D p i$, days post-injury; $A U C$, area under the curve; ELISA, enzyme-linked immunosorbent assay; $T M B$, tetramethylbenzidine dihydrochloride; $G O$, Gene Ontology; KEGG, Kyoto Encyclopedia of Genes and Genomes; GDNF, glial cell line-derived neurotrophic factor; CST, corticospinal tract; $E A U$, experimental autoimmune uveoretinitis

Open Access This article is licensed under a Creative Commons Attribution 4.0 International License, which permits use, sharing, adaptation, distribution and reproduction in any medium or format, as long as you give appropriate credit to the original author(s) and the source, provide a link to the Creative Commons licence, and indicate if changes were made. The images or other third party material in this article are included in the article's Creative Commons licence, unless indicated otherwise in a credit line to the material. If material is not included in the article's Creative Commons licence and your intended use is not permitted by statutory regulation or exceeds the permitted use, you will need to obtain permission directly from the copyright holder. To view a copy of this licence, visit http://creativecommons.org/licenses/by/4.0/.

\section{References}

Anderson KD (2004) Targeting recovery: priorities of the spinal cordinjured population. J Neurotrauma 21(10):1371-1383

Carelli S, Marfia G, Di Giulio AM, Ghilardi G, Gorio A (2011) Erythropoietin: recent developments in the treatment of spinal cord injury. Neurol Res Int 2011:453179

Carelli S, Giallongo T, Gombalova Z, Merli D, Di Giulio AM, Gorio A (2017) EPO-releasing neural precursor cells promote axonal regeneration and recovery of function in spinal cord traumatic injury. Restor Neurol Neurosci 35(6):583-599

Celik M, Gökmen N, Erbayraktar S, Akhisaroglu M, Konakc S, Ulukus C, Genc S, Genc K, Sagiroglu E, Cerami A, Brines M (2002) Erythropoietin prevents motor neuron apoptosis and neurologic disability in experimental spinal cord ischemic injury. Proc Natl Acad Sci U S A 99(4):2258-2263

Chisholm SP, Cervi AL, Nagpal S, Lomax AE (2012) Interleukin-17A increases neurite outgrowth from adult postganglionic sympathetic neurons. J Neurosci 32(4):1146-1155

Cho YK, Kim G, Park S, Sim JH, Won YJ, Hwang CH, Yoo JY, Hong HN (2012) Erythropoietin promotes oligodendrogenesis and myelin repair following lysolecithin-induced injury in spinal cord slice culture. Biochem Biophys Res Commun 417(2):753-759

Christian P, Franz-Josef K, Stephan N (2013) Interleukin-1beta (IL-1 $\beta$ ) in the peripheral blood of dogs as a possible marker for the detection of early stages of inflammation. Open J Vet Med 3:302-308. https:// doi.org/10.4236/ojvm.2013.37049

Claus-Walker J, Dunn CD (1984) Spinal cord injury and serum erythropoietin. Arch Phys Med Rehabil 65(7):370-374

Costa DD, Beghi E, Carignano P, Pagliacci C, Faccioli F, Pupillo E, Messina P, Gorio A, Redaelli T (2015) Tolerability and efficacy of erythropoietin (EPO) treatment in traumatic spinal cord injury: a preliminary randomized comparative trial vs. methylprednisolone (MP). Neurol Sci 36(9):1567-1574

Dalgaard L (2015) Comparison of minipig, dog, monkey and human drug metabolism and disposition. J Pharmacol Toxicol Methods 74:80-92

Foley LS, Fullerton DA, Mares J, Sungelo M, Weyant MJ, Cleveland JC et al (2017) Erythropoietin's beta common receptor mediates neuroprotection in spinal cord neurons. Ann Thorac Surg 104(6):1909-1914

Fujiyoshi T, Kubo T, Chan CC, Koda M, Okawa A, Takahashi K et al (2010) Interferon- $\gamma$ decreases chondroitin sulfate proteoglycan expression and enhances hindlimb function after spinal cord injury in mice. J Neurotrauma 27(12):2283-2294

Gerasimenko YP, Lu DC, Modaber M, Zdunowski S, Gad P, Sayenko DG, Morikawa E, Haakana P, Ferguson AR, Roy RR, Edgerton VR (2015) Noninvasive reactivation of motor descending control after paralysis. J Neurotrauma 32(24):1968-1980

Gorio A, Madaschi L, Di Stefano B, Carelli S, Di Giulio AM, De Biasi S, Coleman T, Cerami A, Brines M (2005) Methylprednisolone neutralizes the beneficial effects of erythropoietin in experimental spinal cord injury. Proc Natl Acad Sci U S A 102(45):16379-16384 
Grasso G, Sfacteria A, Passalacqua M, Morabito A, Buemi M, Macrì B, Brines ML, Tomasello F (2005) Erythropoietin and erythropoietin receptor expression after experimental spinal cord injury encourages therapy by exogenous erythropoietin. Neurosurgery 56(4):821-827

Habash T, Saleh A, Roy Chowdhury SK, Smith DR, Fernyhough P (2015) The proinflammatory cytokine, interleukin-17A, augments mitochondrial function and neurite outgrowth of cultured adult sensory neurons derived from normal and diabetic rats. Exp Neurol 273:177-189

Hicks KE, Zhao Y, Fallah N, Rivers CS, Noonan VK, Plashkes T, Wai EK, Roffey DM, Tsai EC, Paquet J, Attabib N, Marion T, Ahn H, Phan P, Network RHSCIR (2017) A simplified clinical prediction rule for prognosticating independent walking after spinal cord injury: a prospective study from a Canadian multicenter spinal cord injury registry. Spine J 17(10):1383-1392

Hong HN, Shim JH, Won YJ, Yoo JY, Hwang CH (2018) Therapeutic time window for the effects of erythropoietin on astrogliosis and neurite outgrowth in an in vitro model of spinal cord injury. Medicine (Baltimore) 97(9):e9913

Hu MH, Zheng QF, Jia XZ, Li Y, Dong YC, Wang CY, Lin QY, Zhang FY, Zhao RB, Xu HW, Zhou JH, Yuan HP, Zhang WH, Ren H (2014) Neuroprotection effect of interleukin (IL)-17 secreted by reactive astrocytes is emerged from a high-level IL-17-containing environment during acute neuroinflammation. Clin Exp Immunol 175(2):268-284

Hulme CH, Brown SJ, Fuller HR, Riddell J, Osman A, Chowdhury J, Kumar N, Johnson WE, Wright KT (2017) The developing landscape of diagnostic and prognostic biomarkers for spinal cord injury in cerebrospinal fluid and blood. Spinal Cord 55(2):114-125

Ishii H, Jin X, Ueno M, Tanabe S, Kubo T, Serada S, Naka T, Yamashita T (2012) Adoptive transfer of Th1-conditioned lymphocytes promotes axonal remodeling and functional recovery after spinal cord injury. Cell Death Dis 3:e363

Ishii H, Tanabe S, Ueno M, Kubo T, Kayama H, Serada S, Fujimoto M, Takeda K, Naka T, Yamashita T (2013) ifn- $\gamma$-dependent secretion of IL-10 from Th1 cells and microglia/macrophages contributes to functional recovery after spinal cord injury. Cell Death Dis 4:e710

Jeffery ND, Lakatos A, Franklin RJ (2005) Autologous olfactory glial cell transplantation is reliable and safe in naturally occurring canine spinal cord injury. J Neurotrauma 22(11):1282-1293

Kaminski L, Virginie C, Eduard C, Kouame M, Mac-Thiong JM (2017) Functional outcome prediction after traumatic spinal cord injury based on acute clinical factors. J Neurotrauma 34(12):2027-2033

Kijima K, Kubota K, Hara M, Kobayakawa K, Yokota K, Saito T, Yoshizaki S, Maeda T, Konno D, Matsumoto Y, Nakashima Y, Okada S (2019) The acute phase serum zinc concentration is a reliable biomarker for predicting the functional outcome after spinal cord injury. EBioMedicine 41:659-669

Krishna V, Andrews H, Varma A, Mintzer J, Kindy MS, Guest J (2014) Spinal cord injury: how can we improve the classification and quantification of its severity and prognosis? J Neurotrauma 31(3):215-227

Ksiazek-Winiarek D, Szpakowski P, Turniak M, Szemraj J, Glabinski A (2017) IL-17 exerts anti-apoptotic effect via miR-155-5p downregulation in experimental autoimmune encephalomyelitis. J Mol Neurosci 63(3-4):320-332

Kumar R, Lim J, Mekary RA, Rattan A, Dewan MC, Sharif SY, OsorioFonseca E et al (2018) Traumatic spinal injury: global epidemiology and worldwide volume. World Neurosurg 113:e345-e363

Kunis G, Baruch K, Rosenzweig N, Kertser A, Miller O, Berkutzki T, Schwartz M (2013) IFN- $\gamma$-dependent activation of the brain's choroid plexus for CNS immune surveillance and repair. Brain 136(Pt 11):3427-3440

Kwon BK, Hillyer J, Tetzlaff W (2010) Translational research in spinal cord injury: a survey of opinion from the SCI community. J Neurotrauma 27(1):21-33
Kwon BK, Bloom O, Wanner IB, Curt A, Schwab JM, Fawcett J, Wang KK (2019) Neurochemical biomarkers in spinal cord injury. Spinal Cord 57(10):819-831

Matis GK, Birbilis TA (2009) Erythropoietin in spinal cord injury. Eur Spine J 18(3):314-323

Moore SA, Granger N, Olby NJ, Spitzbarth I, Jeffery ND, Tipold A, Nout-Lomas YS, da Costa RC, Stein VM, Noble-Haeusslein LJ, Blight AR, Grossman RG, Basso DM, Levine JM (2017) Targeting translational successes through CANSORT-SCI: using pet dogs to identify effective treatments for spinal cord injury. J Neurotrauma 34(12):2007-2018

Moynes DM, Vanner SJ, Lomax AE (2014) Participation of interleukin 17A in neuroimmune interactions. Brain Behav Immun 41:1-9

Olby NJ, De Risio L, Muñana KR, Wosar MA, Skeen TM, Sharp NJ et al (2001) Development of a functional scoring system in dogs with acute spinal cord injuries. Am J Vet Res 62(10):1624-1628

Raineteau O, Schwab ME (2001) Plasticity of motor systems after incomplete spinal cord injury. Nat Rev Neurosci 2(4):263-273

Rasmussen R, Carlsen EM (2016) Spontaneous functional recovery from incomplete spinal cord injury. J Neurosci 36(33):8535-8537

Rodrigues LF, Moura-Neto V, Spohr E, TCLS (2018) Biomarkers in spinal cord injury: from prognosis to treatment. Mol Neurobiol 55(8):6436-6448

Simon F, Scheuerle A, Gröger M, Vcelar B, McCook O, Möller P, Georgieff M, Calzia E, Radermacher P, Schelzig H (2011) Comparison of carbamylated erythropoietin-FC fusion protein and recombinant human erythropoietin during porcine aortic balloon occlusion-induced spinal cord ischemia/reperfusion injury. Intensive Care Med 37(9):1525-1533

Simon FH, Erhart P, Vcelar B, Scheuerle A, Schelzig H, Oberhuber A (2016) Erythropoietin preconditioning improves clinical and histologic outcome in an acute spinal cord ischemia and reperfusion rabbit model. J Vasc Surg 64(6):1797-1804

Victório SC, Havton LA, Oliveira AL (2010) Absence of IFN $\gamma$ expression induces neuronal degeneration in the spinal cord of adult mice. J Neuroinflammation 7:77

Vitellaro-Zuccarello L, Mazzetti S, Madaschi L, Bosisio P, Fontana E, Gorio A, De Biasi S (2008) Chronic erythropoietin-mediated effects on the expression of astrocyte markers in a rat model of contusive spinal cord injury. Neuroscience 151(2):452-466

Wang C, Lv H, Li Q, Gong K, Yang LL, Wei Z, Pan Y, Wang M (2019) RNA sequencing of peripheral blood revealed that the neurotropic TRK receptor signaling pathway shows apparent correlation in recovery following spinal cord injury at small cohort. J Mol Neurosci 68(2):221-233

Yang L, Jones NR, Blumbergs PC, Van DHeuvel C, Moore EJ, Manavis J et al (2005) Severity-dependent expression of pro-inflammatory cytokines in traumatic spinal cord injury in the rat. J Clin Neurosci 12(3):276-284

Yokobori S, Zhang Z, Moghieb A, Mondello S, Gajavelli S, Dietrich WD, Bramlett H, Hayes RL, Wang M, Wang KK, Bullock MR (2015) Acute diagnostic biomarkers for spinal cord injury: review of the literature and preliminary research report. World Neurosurg 83(5):867-878

Yousefifard M, Sarveazad A, Babahajian A, Baikpour M, Shokraneh F, Vaccaro AR, Harrop JS, Fehlings MG, Hosseini M, RahimiMovaghar V (2019) Potential diagnostic and prognostic value of serum and cerebrospinal fluid biomarkers in traumatic spinal cord injury: a systematic review. J Neurochem 149(3):317-330

Publisher's Note Springer Nature remains neutral with regard to jurisdictional claims in published maps and institutional affiliations. 